\title{
EL PERFIL DEL VISITANTE EN SANTIAGO DE COMPOSTELA: TRADICIÓN Y ACTUALIDAD
}

\author{
Yamilé Pérez Guilarte \\ Rubén Camilo Lois González. \\ Universidad de Santiago de Compostela
}

\section{RESUMEN}

Este trabajo estudia el perfil del visitante en Santiago de Compostela y lo compara con el de otros destinos culturales del mundo. Se tomó como base la metodología de ATLAS y se realizaron 400 encuestas a visitantes. Se aprecia el perfil del tradicional turista cultural de edad madura que viaja por vacaciones. Experimenta más motivación por el ambiente del lugar que por el aporte de conocimiento. Asimismo, considera que la arquitectura histórica es el mayor atractivo de Santiago, aunque muestra interés por actividades culturales que impliquen una experiencia más creativa y participativa.

Palabras clave: Perfil del visitante; Santiago de Compostela; oferta cultural creativa.

\section{Visitors' profile in Santiago de Compostela: tradition and present}

\section{ABSTRACT}

The aim of this study is to determine Santiago de Compostela's visitors profile and compare it with those of other cultural destinations worldwide. Based on ATLAS methodology and after 400 visitors were interviewed, the results reveal a traditional middleaged cultural tourist who travels for vacation. It's more motivated by the atmosphere of the place than the actual acquisition of knowledge. Although historical architecture remains to be considered Santiago's main asset, the study shows an increasing interest in cultural activities that provide more creative and interactive experiences.

Keywords: Visitors' profile; Santiago de Compostela; Creative Cultural Supply.

Recibido: 28 de marzo de 2015

Devuelto para su revisión: 19 de mayo de 2015

Aceptado: 15 de junio de 2015

Departamento de Geografía. Universidad de Santiago de Compostela. Praza da Universidade, 1. 15782 SANTIAGO DE COMPOSTELA (España).E-mail: yamypguilarte@gmail.com,rubencamilo.lois@usc.es 


\section{INTRODUCCIÓN}

Santiago de Compostela es un importante destino cultural, no sólo por su condición de ciudad Patrimonio de la Humanidad sino por ser desde épocas medievales la meta del Camino de Santiago, una de las principales rutas de peregrinación de la cristiandad. Posee una imagen internacional consolidada en torno al patrimonio religioso, aunque en los últimos años se aprecia una voluntad por parte de los gestores turísticos de promocionar otras alternativas como el turismo gastronómico, el de congresos y eventos, o el idiomático. Santiago de Compostela es el octavo destino más competitivo entre los 20 más exitosos de España (Exceltur, 2013). Sin embargo, para mantener la competitividad en el actual escenario turístico global es necesario revitalizar los destinos de acuerdo con las nuevas necesidades de los visitantes.

El conocimiento del perfil de las personas que visitan un destino posibilita no sólo orientar la oferta a sus expectativas, sino también personalizar las acciones de publicidad y comercialización en los segmentos de interés. Para la comprensión de la demanda y los atributos del mercado es clave el conocimiento de las características demográficas, que tienen en cuenta aspectos sociales y socio-económicos como: la edad, el género, el empleo, el estatus marital, la educación y los ingresos. Asimismo, se hace imprescindible la comprensión de aspectos relacionados con la forma en que los visitantes organizan su viaje, las motivaciones para viajar al destino, las actividades de mayor preferencia, la imagen que tienen de la ciudad, y su satisfacción con la experiencia turística.

En este sentido, la Association for Tourism and Leisure Education (ATLAS) constituye un referente a nivel mundial por su afán de profundizar en el estudio de los visitantes en destinos culturales. El programa ${ }^{1}$ desarrollado comenzó en Europa y con la colaboración de diversas instituciones e investigadores se ha ampliado a países en todo el mundo, permitiendo realizar más de 40.000 encuestas (ATLAS, 2015). En cada destino éstas pueden ser realizadas en la ciudad como conjunto o en lugares específicos como museos, sitios históricos o festivales. Se establece un mínimo de 200 cuestionarios por destino, que por lo general son respondidos por los propios visitantes porque se disponen de ejemplares traducidos en más de una docena de idiomas. Se entrevistan a los visitantes de forma aleatoria y se incluyen a los procedentes de la región, a los del resto del país, y a los extranjeros. Asimismo, en la selección de la muestra se tienen en cuenta tanto a los turistas como a los excursionistas (Richards y Queirós, 2005). El propósito de este proyecto es lograr un acercamiento al perfil del turista cultural, aunque es claro que algunos aspectos están condicionados por las características de cada destino en cuestión.

El objetivo de este artículo es conocer el perfil del visitante que acude a Santiago de Compostela y compararlo con el de otros destinos culturales del mundo, tomando como base los resultados de ATLAS. Asimismo, se profundiza en el interés de los visitantes por actividades culturales que implican una participación más activa en la experiencia turística. Las mismas podrían enmarcarse en lo que se ha venido denominando turismo creativo (Richards y Raymond, 2000; UNESCO, 2006), y que abarca actividades como la

1 La Asociación ATLAS desarrolló en 1991 el Programa «ATLAS Tourism Research Project. A Global Network for Cultural Tourism Research» con el objetivo de conocer más sobre las motivaciones, el perfil, el comportamiento y las actitudes de los turistas culturales (ATLAS, 2014). 
realización de un taller de gastronomía o la visita a un proceso de producción artesanal. El turismo creativo engloba la creación de experiencias y la implicación activa de los clientes en la producción de esas experiencias (Wurzburguer et al., 2008). Más allá de los elementos que han sido previamente introducidos en circuitos de turismo cultural, lo que los turistas creativos consumen es el modo de vida local, o sea, la vida cotidiana (Richards, 2012). En realidad se trata de consumir la cultura, pero de una forma más participativa (interacción, aprendizaje, elaboración). Al respecto, este artículo plantea conocer de qué forma se expresa éste fenómeno en las personas que visitan Santiago de Compostela.

\section{METODOLOGÍA}

Considerando la relevancia de las investigaciones desarrolladas por ATLAS en el estudio de la demanda cultural, para la realización de este trabajo se partió de la misma base metodológica. Además de tratarse de un cuestionario ya validado, su utilización permitió la comparación de los resultados obtenidos en este estudio con los de ATLAS u otros investigadores que lo han empleado. Para adecuarlo a los objetivos de esta investigación se realizó una adaptación de la última versión para Europa publicada por ATLAS en 2007 (www.tram-research.com/atlas/survey2007.htm). Uno de los cambios propuestos en el cuestionario es la separación del turismo creativo del educativo al estudiar las motivaciones, lo cual permitió profundizar en la magnitud que tiene el turismo creativo en el destino. En este sentido, también se incorporó una pregunta nominal cerrada de respuesta múltiple para conocer en qué medida los visitantes realizaban actividades creativas y cuáles eran las de mayor popularidad, así como conocer la relación entre este grupo de visitantes y los que clasificaron sus vacaciones como creativas. Entre las actividades creativas que se incluyeron se encuentran: talleres de gastronomía; cenas a ciegas; catas y degustaciones; talleres de música, pintura, artesanía, literatura; y visitas a talleres de artesanos o actividades productivas. De igual modo, se incorporó una pregunta que ya había sido contemplada en la versión de ATLAS 2004, relacionada con la autenticidad de la imagen de la ciudad y de sus atractivos culturales.

El universo objeto de estudio lo integran los visitantes a Santiago de Compostela, tanto turistas como excursionistas, residentes en Galicia, en el resto de España o en el extranjero. Los peregrinos no fueron seleccionados para este estudio. En la actualidad el fenómeno de la peregrinación y el turismo son cada vez más convergentes, debido a que la antigua motivación religiosa ha perdido protagonismo y coexiste con motivaciones seculares, apareciendo los peregrinos turistas (Lois y López, 2012). Sin embargo, de manera general los peregrinos presentan un perfil diferente al del resto de visitantes, por la forma de organización del viaje y por su comportamiento en la ciudad. Por ejemplo, viajan menos en familia, en muchas ocasiones no pernoctan o permanecen por menos tiempo, se alojan en albergues o pensiones, realizan menos compras; por lo que su gasto en el destino es inferior (Santos, 2006; Santos y Lois, 2011). Asimismo, hay que mencionar que el volumen de peregrinos que recibe la ciudad en realidad es una pequeña parte del total de visitantes (Santos, 2006), si bien es cierto que constituyen un elemento de singularidad del destino. Estas razones motivaron la decisión de excluir a los peregrinos de la muestra, evitando así un sesgo que imposibilitara la comparación de los resultados con otros destinos culturales 
del mundo que no son de peregrinación. En definitiva, se excluyó un segmento específico con bajo peso en el total de visitantes, pero que en cambio podría sesgar la muestra para el objetivo propuesto.

Las encuestas se realizaron durante los meses de julio y agosto de 2013, por ser un período de gran afluencia de visitantes. No obstante, fueron distribuidas de forma tal que se realizaran tanto los fines de semana como durante la misma. Se estableció un nivel de error de un 4,9\% y para un $95 \%$ de confianza se estimó el tamaño de la muestra en 400 . Las personas fueron entrevistadas a través de un muestreo aleatorio simple. Al interceptar a los individuos se les preguntaba si su motivo principal de visita a la ciudad era el peregrinaje, y sólo si respondían negativamente se procedía a realizar la encuesta. La mayor parte de los entrevistados prefirió responder por su cuenta el cuestionario (95\%), lo cual fue posible debido a que estaba traducido en cinco idiomas: español, inglés, italiano, francés y portugués. En el caso de las personas que viajaban en grupo sólo se realizó un cuestionario, eligiendo a la persona que estuviera más próxima a cumplir años.

Los principales atractivos turísticos de la ciudad son: la catedral y su entorno, el patrimonio histórico-artístico y el conjunto de la ciudad histórica (Observatorio Turístico de Santiago, 2014). Teniendo en cuenta este hecho, se estableció la zona vieja y su entorno como área para la realización del trabajo de campo (Figura 1). En este contexto se entrevis-

\section{Figura 1}

\section{LOCALIZACIÓN DE LOS SITIOS DONDE SE REALIZARON LAS ENCUESTAS}

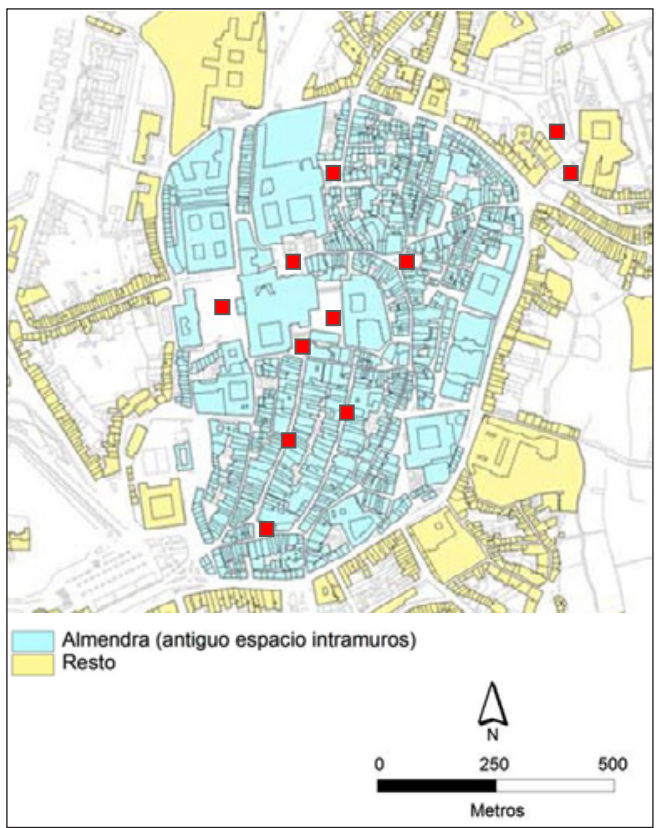

Fuente: Aldrey, Santos, y Pazos (2009). 
taron tanto a individuos que paseaban por las calles o descansaban en plazas (65\%), como aquellos que visitaban un museo, un edificio histórico, una exposición cultural (21\%), o disfrutaban del tren turístico $(14 \%)$.

El procesamiento estadístico de los resultados se realizó a través del Programa SPSS (Statistical Package for the Social Sciences, versión 18). Una vez finalizada la etapa de introducción de la información, se realizó un análisis descriptivo e inferencial para presentar y explicar los resultados. Asimismo, se establecieron comparaciones con investigaciones realizadas por ATLAS u otros autores en diferentes destinos culturales del mundo, así como con los resultados del Observatorio Turístico de Santiago de Compostela.

\section{RESULTADOS}

La presentación de los resultados está estructurada en cuatro apartados. En primer lugar se define el perfil sociodemográfico del conjunto de individuos encuestados. A continuación se presentan las motivaciones que tuvieron para realizar el viaje, la forma de organizarlo y las fuentes de información consultadas antes de viajar y una vez en el destino. Por último, se exponen las principales actividades culturales realizadas, el nivel de satisfacción y los aspectos vinculados con la imagen de la ciudad.

\subsection{Perfil sociodemográfico}

E1 89\% de los visitantes encuestados fueron turistas, es decir, pernoctaron al menos una noche en el destino, cifra superior al 70\% registrado por los estudios de ATLAS (Richards y Queirós, 2005). Como muestra la Figura 2, la mayor parte de los mismos fueron españoles precedentes de otras partes de España. La proporción de extranjeros entrevistados para este trabajo estuvo por debajo del $42 \%$ obtenido por ATLAS (Richards y Queirós, 2005). Este comportamiento se explica en el hecho de que el turismo interno tiene una gran repercusión en Santiago de Compostela. De este modo, el mercado español representa más del $50 \%$ de los visitantes, incluso en ocasiones llegando a alcanzar valores cercanos a 70\% (Observatorio Turístico de Santiago, 2014).

\section{Figura 2 \\ PROCEDENCIA DE LOS ENTREVISTADOS}

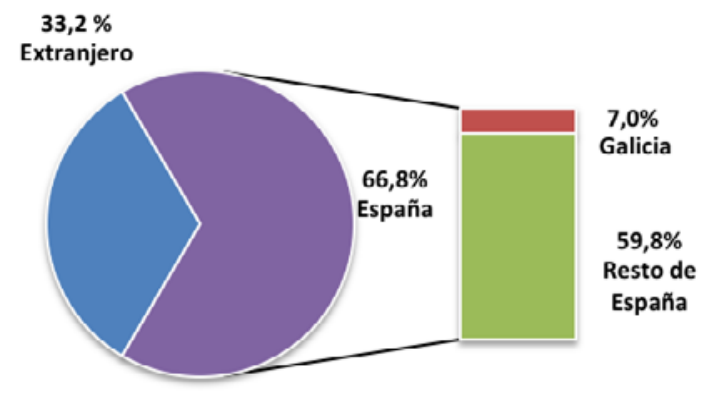

Cuadernos de Turismo, 37, (2016), 305-322 
Los visitantes extranjeros procedieron de 27 países principalmente de Europa y América. Los países más representados son Francia e Italia con el 14 y $10 \%$ respectivamente. Seguidamente, con alrededor de un $8 \%$ se encuentran Portugal, Reino Unido y Alemania. Este resultado se corresponde con los principales mercados emisores de visitantes a la ciudad según información del Observatorio Turístico de Santiago, aunque los alemanes tienen un mayor protagonismo y el mercado portugués está más asociado al excursionismo (Observatorio Turístico de Santiago, 2014). En cuanto a América, los países más relevantes son Estados Unidos, México y Brasil.

El 55\% de los encuestados fueron mujeres y el $45 \%$ hombres, distribución que corresponde con el perfil del visitante cultural de ATLAS, donde el 53\% son del sexo femenino (Richards, 2008). También coincide con otros trabajos, por ejemplo, Isaac (2008) en su estudio de los visitantes culturales a diferentes ciudades holandesas revela un $54 \%$ de mujeres. Mientras que, Hernández-Mogollón et al. (2013), manifiestan la presencia de un $53 \%$ en su estudio del perfil del turista cultural en Plasencia.

Con relación a la edad, se observa el tradicional turista cultural de edad madura. La Figura 3 muestra que los mayores de 60 años representaron un 12\% y que casi el $60 \%$ tenían 40 años o más. Esta última cifra es coherente con el 58\% de Isacc (2008), aunque más alejado del 47\% de Hernández-Mogollón et al. (2013). Mientras que, los visitantes entre 20 y 29 años fueron el 18\%. Aunque similar al trabajo de Isaac (2008), esta cifra está bastante por debajo de los resultados de ATLAS, donde los jóvenes representan un $30 \%$ (Richards, 2008).

Según datos del Observatorio de Turismo de Santiago, el porcentaje de visitantes mayores de 60 años se ha incrementado. De estar entre 6 y 11\% entre 2005 y 2010 a alcanzar $16 \%$ en 2011 y $15 \%$ en 2012. Los menores de 30 años han disminuido, de porcentajes alrededor de 40\% entre 2007 y 2009 pasó a valores entre 20 y 30\% en 2011 y 2012 respectivamente (Observatorio Turístico de Santiago, 2014). Por tanto, parece ser que en Santiago no se observa la tendencia que indica ATLAS sobre visitantes culturales más jóvenes. Este hecho podría tener relación con la imagen mediática, promocional y artística de Santiago de Compostela alrededor de estereotipos como su catedral, su centro histórico y el Camino de Santiago (Escudero, 2013), atrayendo a un segmento de visitantes de mayor edad. De igual modo, podría influir la falta de consolidación de una oferta creativa con capacidad de atracción al público joven. Este es un aspecto significativo teniendo en cuenta que este segmento va teniendo un mayor peso en los viajes a nivel mundial, representando un 20\% (OMT, 2011). Como tal, los jóvenes exigen una oferta acorde a sus expectativas a través de experiencias más entretenidas y que permitan interactuar con la población local, siendo esta última la motivación más importante para ellos (OMT, 2011).

Hay que destacar el alto nivel educativo de los entrevistados, el 62\% tenían un nivel de Licenciado, Máster o Doctorado. Este resultado coincide con el perfil del turista cultural de ATLAS de casi un 70\% (Richards, 2008), confirmando el crecimiento de la educación superior en el conjunto de la población, y en consecuencia de las personas que visitan los sitios culturales. Asimismo, el 75\% afirmó encontrarse en situación favorable de empleo, de ellos el 58\% como empleados y el 17\% como autónomos. La proporción de jubilados y estudiantes es prácticamente similar. Mientras que los desempleados representaron un $5 \%$, lógicamente un porcentaje bajo teniendo en cuenta las limitaciones en el consumo que 
tienen las personas sin empleo. Con relación a las categorías de ocupación, más del 70\% de los entrevistados fueron directivos o profesionales. Asimismo, el 27\% consideró que su ocupación estaba relacionada con la cultura, resultado similar al de ATLAS (30\%), quien además señala un incremento con respecto a años anteriores. Este comportamiento refleja una expansión de las profesiones culturales y creativas, o de lo que se reconoce como este tipo de profesiones, y de aquellos involucrados en las mismas que visitan destinos culturales (Richards, 2008).

Figura 3

\section{INTERVALOS DE EDAD DE LOS ENTREVISTADOS}

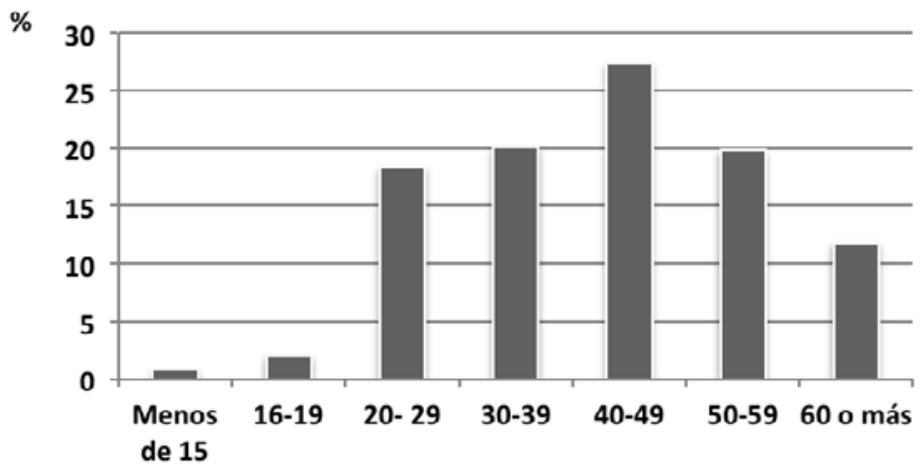

\subsection{Motivación y organización del viaje}

Los visitantes entrevistados mostraron una alta fidelidad, pues el $47 \%$ de ellos ya habían realizado visitas anteriores. Como en la mayoría de los destinos, la principal motivación son las vacaciones, elegidas por el $73 \%$ de los encuestados (Figura 4). Como se aprecia, el $15 \%$ acudió a Santiago para visitar un atractivo cultural y el 1\% para asistir a un evento, con lo cual un $16 \%$ de visitantes viajaron a Santiago por un motivo cultural. Si se analizan sólo a los que pernoctaron, es decir, a los turistas, también coincide este mismo porcentaje. Este conjunto de turistas formarían parte de la categoría de turista motivado culturalmente, según la clasificación de McKercher y Du Cros (2002).

De igual modo, el $16 \%$ de turistas motivados por la cultura obtenido en este estudio se acerca al 12\% aportado por el Ministerio de Educación, Cultura y Deporte (2013) para el caso de España. Asimismo, es coherente con los resultados de McKercher y Du Cros (2003) para los visitantes de Hong Kong, donde este grupo alcanzó el 13\%. Por tanto, hay que destacar que la mayoría de los turistas que se dirigen a grandes ciudades y destinos patrimoniales viajan motivados por razones recreativas, más que por una experiencia profunda de aprendizaje.

Las visitas a amigos y familiares ocuparon el $4 \%$ de las motivaciones, un resultado de esperar considerando que cada vez más las personas tienen en cuenta a los conocidos a la hora de elegir un destino. Evidentemente, la posibilidad de obtener alojamiento gratis se ha convertido en un incentivo, y más aún en períodos de recesión económica. Esta moda- 


\section{Figura 4 \\ MOTIVO PARA LA REALIZACIÓN DEL VIAJE DE LOS ENCUESTADOS}

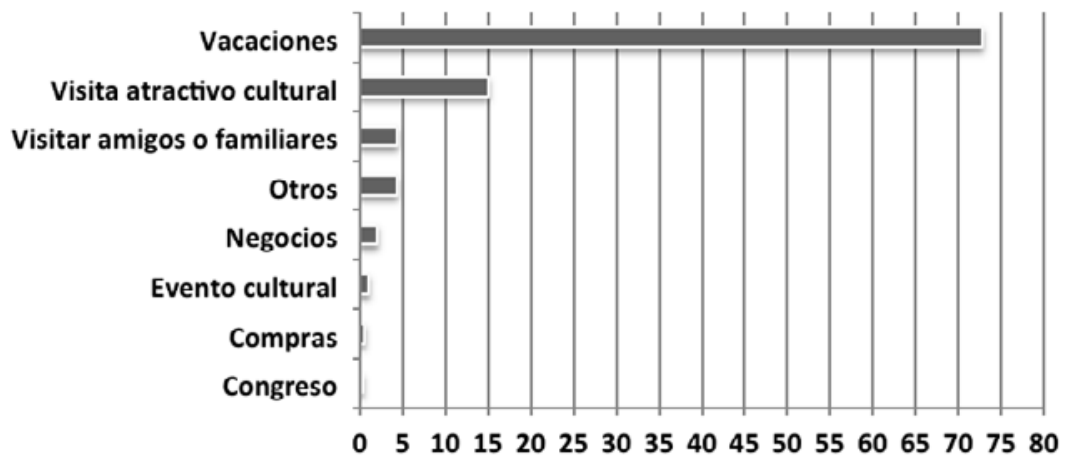

$\%$

lidad de viaje (Visiting Friends and Relatives, VFR) ha crecido en importancia, aunque según Backer (2007) no ha sido suficientemente estudiada, en parte por estar asociada a un bajo impacto económico. Asimismo, el $4 \%$ no se sintió identificado con ninguno de los motivos presentados en la encuesta. El 2\% realizó el viaje por negocios, mientras que prácticamente no hubo visitantes cuya motivación principal fuera asistir a un congreso o realizar compras, quizás por la época del año en que se realizaron las entrevistas.

En el informe de ATLAS, coinciden las tres primeras motivaciones resultantes de este estudio. Las vacaciones como principal motivo, aunque con menor porcentaje (56\%), seguidas de las visitas a un atractivo cultural, ligeramente superior (alrededor del 18\%); y las visitas a amigos y familiares, también con mayor valor (cercanas al 10\%). Otras similitudes fueron el 5\% que manifestó otros motivos, y el hecho de que el turismo de congresos y el deportivo estén en últimas posiciones. Sin embargo, se aprecia una diferencia con relación a la participación en eventos culturales, aspecto que superó el 5\% de los encuestados en el estudio de ATLAS (Richards, 2008).

Hay que mencionar que en el procesamiento de las encuestas 13 fueron desestimadas porque las personas eligieron más de un motivo o tipo de vacaciones. Por tanto, para estudios posteriores habría que considerar la posibilidad de que se pudiera reflejar una motivación combinada. Por citar un ejemplo, podría establecerse como motivos principales vacaciones, y visitar a amigos y familiares; o en cuanto al tipo de vacaciones, estas podrían ser culturales, y de sol y playa. Evidentemente, esto complicaría la clasificación de los visitantes en una modalidad turística, pero se trata de contemplar una situación que está aconteciendo.

Entre los visitantes cuya motivación para viajar al destino fue las vacaciones destacan las culturales, como es de esperar en un destino como Santiago de Compostela (Figura 5). El $43 \%$ de los que estaban de vacaciones clasificaron su viaje como cultural, representando un $32 \%$ del total de entrevistados. El turismo itinerante ocupa el segundo puesto (21\%), abarcando un $16 \%$ del total. Este resultado muestra que las personas organizan viajes que 


\section{Figura 5 \\ TIPO DE VACACIONES REALIZADAS POR LOS ENTREVISTADOS}

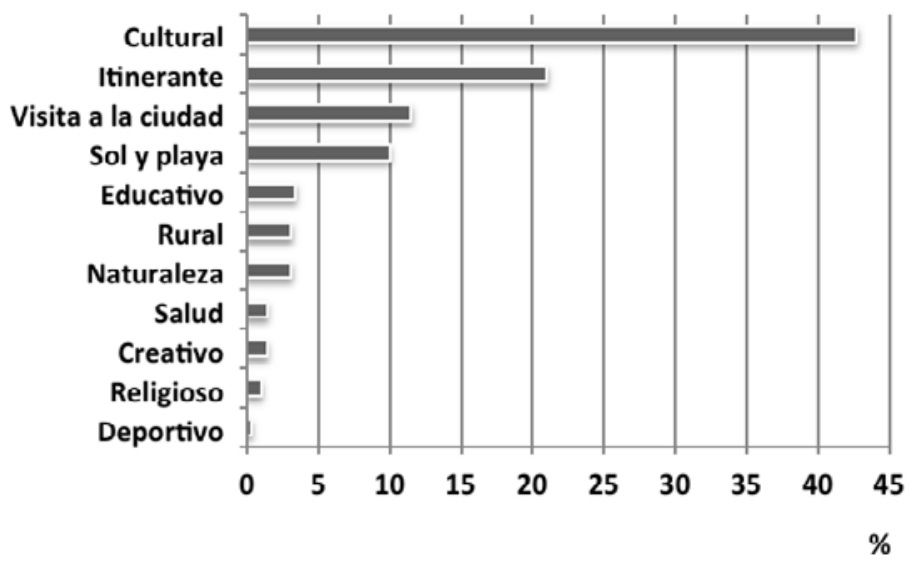

aunque con menor estancia, les permitan visitar un mayor número de sitios. Este comportamiento es congruente con las necesidades del turista actual, fundamentadas en la búsqueda de entretenimiento y diversión (OMT, 2011; Pérez Guilarte, 2014).

La visita a la ciudad fue el tercer motivo vacacional (12\% de este grupo). El turismo de sol y playa fue el cuarto entre los que estaban de vacaciones (10\%), evidentemente debido a que el estudio se realizó durante el verano y a que existen un conjunto de playas en las cercanías de Santiago. El turismo educativo, de naturaleza y rural estuvieron representados de forma pareja con valores alrededor del 3\%. En última posición y con escasa participación entre los que estaban de vacaciones, aparecen con alrededor del $1 \%$, el turismo creativo, el religioso y el de salud; y con $0,3 \%$ el deportivo.

Las cuatro primeras tipologías de vacaciones obtenidas en este estudio coinciden con los resultados de ATLAS. Se observa también similitud con respecto a los porcentajes de las visitas itinerantes (alrededor de $22 \%$ ) y de las vacaciones de playa (en torno a $7 \%$ ). No obstante, se aprecian discrepancias en las vacaciones culturales (sobre 33\%) y la visita a la ciudad (21\% aproximadamente). El turismo creativo y educativo, que en la encuesta original aparecen en el mismo ítem, engloban a menos del 5\% de los vacacionistas, resultado similar al de este estudio $(4,8)$ si se suman estas dos modalidades (Richards, 2008).

La Figura 6 presenta el porcentaje de entrevistados que manifestaron estar de acuerdo con algunos aspectos relacionados con la motivación. El mayor interés lo tuvo el disfrute del ambiente del lugar, siendo seleccionado por el $63 \%$ de los encuestados. Asimismo, el hecho de que el sitio disponga de cosas interesantes para ver y que resulte agradable fue atractivo para el 57 y $58 \%$ respectivamente. Mientras que, el parámetro menos valorado fue el aporte de nuevo conocimiento (34\%). Este último también fue el de menor importancia en el informe de ATLAS (Richards y Queirós, 2005; Richards, 2008), con lo cual parece ser que las personas están más interesadas en experimentar un ambiente agradable y con actividades interesantes que realizar, en detrimento de la clásica motivación de aprendizaje. 


\section{Figura 6 \\ ASPECTOS MOTIVACIONALES DEL DESTINO}

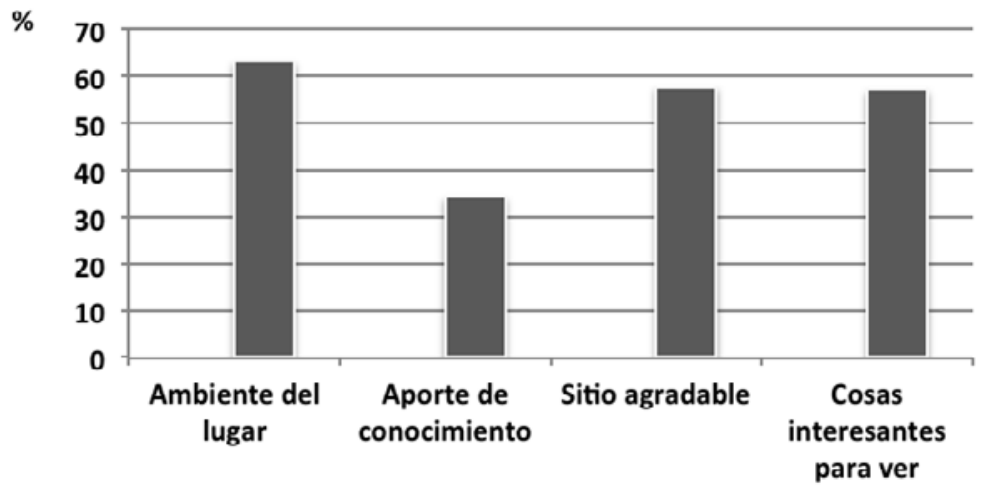

El $67 \%$ de los que pernoctaron en la ciudad eligieron un hotel para su estancia. A continuación le siguieron los apartamentos con casi $10 \%$ y en tercer lugar las casas de familiares y amigos (6\%). Con porcentajes alrededor de $4 \%$ aparecen las pensiones, las segundas residencias y el camping. Las casas rurales fueron elegidas por el 3\%, mientras los albergues juveniles apenas por el 0,8\%. De igual modo, en el estudio de ATLAS fueron los hoteles el alojamiento más empleado, aunque no alcanza el 50\% de los entrevistados. En segundo lugar se ubican las casas de familiares o amigos, con algo más de 10\%; y en tercero las pensiones y apartamentos con apenas $5 \%$. Por su parte, los albergues juveniles tuvieron un mayor protagonismo (alrededor de 5\%), mientras que las segundas residencias fueron el de menor utilización (sobre 1\%) (Richards, 2008).

El viaje es planificado principalmente en familia (44\%) y en pareja (36\%), aunque también de forma individual (9\%) y con amigos (8\%). Sin embargo, los grupos sólo representaron el $2 \%$ del total, posiblemente por la dificultad que implica el acceso a los mismos para la realización de encuestas. Según los datos del Observatorio Turístico de Santiago, los grupos tienen una mayor presencia en la ciudad, constituyendo entre un 5 y $16 \%$ de los visitantes. De igual modo, son inferiores los viajes en familia (entre $10 \mathrm{y}$ $16 \%$ ) y superiores los realizados con amigos (entre 20 y 27\%) (Observatorio Turístico de Santiago, 2014). Posiblemente, el hecho de que el estudio se realizara en verano influyó en que los viajes en familia tuvieran una participación más destacada.

Más del 50\% de los visitantes reservó el viaje y el alojamiento por separado, otros prefirieron no hacer reservas, mientras una pequeña parte escogió un paquete de oferta. Entre los que realizaron reservas más del $70 \%$ lo hizo a través de Internet; y el resto utilizó de forma equitativa una agencia de viajes o el teléfono, fax o email. Este resultado es lógico considerando el continuo incremento de las compras a través de Internet, y en especial en el sector turístico. Este aumento también se evidencia si se compara con el $42 \%$ de usuarios que compraron servicios por Internet registrado por ATLAS en 2007 (Richards, 2008), mostrando cómo se ha incrementado la confianza de los usuarios a la hora de hacer las compras en línea. 
Es interesante el hecho de que a pesar de la masificación del uso de Internet, las agencias de viaje conserven una porción de mercado, aunque sea pequeña, si bien es cierto que han tenido que replantear su estrategia a consecuencia de la gran competencia. Como señalan Oliviera et al. (2013) en su estudio sobre los desafíos de las agencias de viajes, es vital el desarrollo de aspectos claves como la seguridad, la experiencia o la asistencia 24 horas. Estos factores posibilitan que las agencias aún sigan en la preferencia de algunas personas, fundamentalmente para viajes de media y larga duración.

Sin embargo, a la hora de buscar información sobre el destino, las agencias de viajes se ven notablemente desplazadas por otras fuentes (Figura 7). Sólo el $9 \%$ de los entrevistados manifestó haber consultado una agencia antes de viajar, coincidiendo con los valores de ATLAS (10\%). Internet es el medio más empleado, siendo utilizado por el $60 \%$ de las personas entrevistadas, resultado que difiere del proyecto ATLAS (Richards, 2008), donde más que un favoritismo por Internet (38\%), aún se apreciaba un balance con las consultas a amigos y familiares (algo más del 40\%). En la actualidad, se puede decir que las opiniones de otras personas siguen teniendo protagonismo, lo que ocurre es que la búsqueda se ha trasladado a la comunidad cibernauta. Cuando las personas se informan sobre un destino o servicio turístico en Internet, lo que hacen es visitar blogs de viajes para conocer los comentarios de otros que ya visitaron el lugar (Chamorro, 2008; Domínguez y Araújo, 2012). En definitiva, no son amigos o familiares pero sus recomendaciones también van a determinar si escogemos un destino, un hotel o un museo. Tanto en el estudio de ATLAS como en el realizado en este artículo, después de Internet y los amigos y familiares, las fuentes más importantes son las visitas anteriores y las guías de viaje. Mientras que, los folletos de turoperadores, los periódicos, las revistas, la radio, la televisión, y las oficinas de turismo tuvieron un bajo impacto.

Una vez en el destino, los visitantes prefirieron consultar en primera opción los centros de información turística, esta fuente fue escogida por el $39 \%$ de los encuestados, resultado similar al informe de ATLAS (Richards, 2008). Sin embargo, la segunda fuente señalada

\section{Figura 7 \\ FUENTES DE INFORMACIÓN CONSULTADAS ANTES DE VIAJAR}

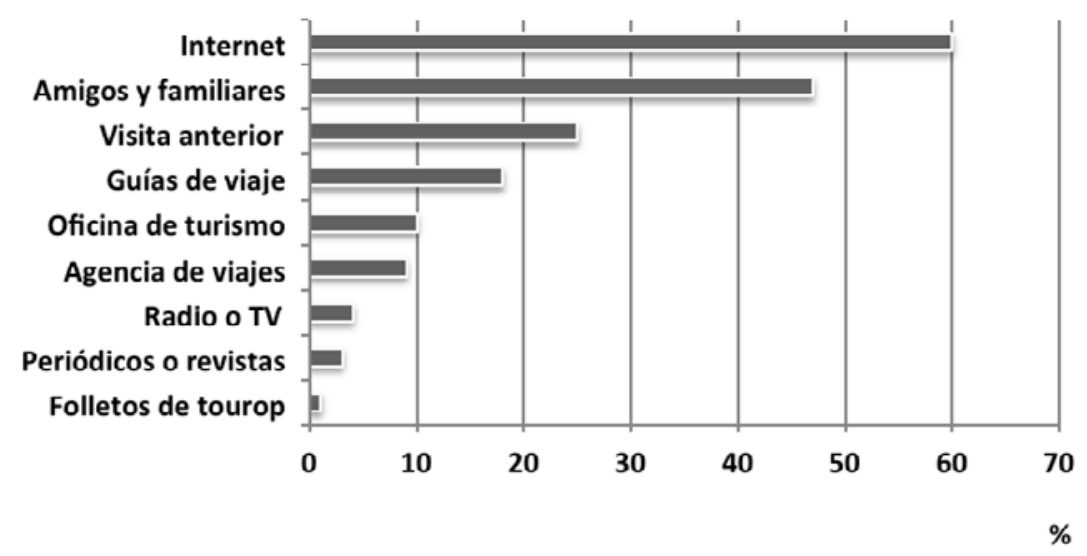


en este estudio fue Internet (33\%), mientras que en ATLAS fue la consulta a los familiares y amigos (menos del 25\%). Esto puede deberse a la facilidad que cada vez más tienen los visitantes para conectarse a través de redes Wifi gratuitas en hoteles, restaurantes y otras instalaciones; así como a la introducción de aplicaciones para dispositivos móviles que brindan información sobre la ciudad o venden servicios turísticos. En ambos casos las fuentes de menor popularidad son: los turoperadores, los periódicos, las revistas, la radio y la televisión.

La estancia promedio de aquellos que pernoctaron fue de 3,46 noches, alcanzando un valor mínimo de 1 y un máximo de 15 , aunque el $75 \%$ permaneció menos de 4 noches. La estancia media en este estudio es superior a las 2,6 noches que indica el Observatorio Turístico de Santiago. La diferencia obtenida podría deberse al hecho de que el trabajo de campo se realizó en los meses de verano. En esta época la complementariedad con otros productos turísticos como la playa o la naturaleza en los alrededores de Santiago pudo incidir en una mayor estancia.

\subsection{Actividades culturales y creativas}

En cuanto a las actividades culturales, los monumentos son la atracción de mayor preferencia (Figura 8). En segundo lugar, y escogidos también por más de la mitad de los visitantes, se encuentran los sitios religiosos, los sitios históricos y los museos. Según los datos del Observatorio Turístico de Santiago visitar los museos tampoco es la actividad de más interés, aunque se aprecia una tendencia creciente. Entre los años 2007 y 2010 sólo entre el 22 y $29 \%$ de los visitantes realizaban esta actividad, en 2011 ascendió hasta un 42\%, y en 2013 a 55\% (según el resultado de este estudio). En cualquier caso, la renovación del Museo de las Peregrinaciones puede estar en el origen de estos cambios. En Santiago hay buenos museos mal publicitados para el turismo y cualquier cambio en la oferta se nota.

\section{Figura 8 \\ ATRACCIONES CULTURALES VISITADAS}

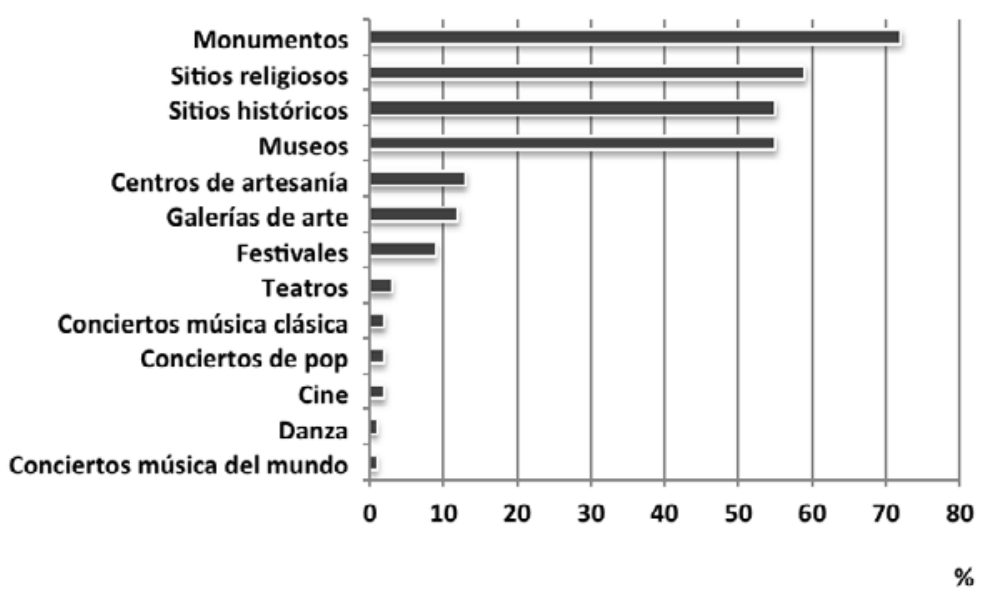


Este patrón de comportamiento cambia con respecto al de ATLAS, puesto que aunque las actividades más escogidas fueron las mismas, el orden es diferente. La actividad de mayor interés es asistir a un museo, señalada por casi el $65 \%$ de los visitantes. En segundo lugar, en vez de los sitios religiosos, son los históricos los más visitados (Richards, 2008). En el caso de Santiago es comprensible debido al ambiente de lugar final de una ruta de peregrinación que se «respira» en la ciudad. De la misma forma, coincide en ambos casos el hecho de que las atracciones de menor preferencia para los visitantes sean: los conciertos de música, el cine y las manifestaciones de danza.

En los estudios de ATLAS el turismo creativo y el educativo aparecen en la encuesta dentro de un mismo ítem. En esta investigación se separaron para poder estudiar la magnitud del turismo creativo sin la interferencia del educativo, que representa en Santiago entre el 2 y $4 \%$ de los visitantes (Observatorio Turístico de Santiago, 2014). El turismo creativo sólo fue seleccionado por el 1,4\% de los entrevistados, aunque el $23 \%$ de los entrevistados (90 individuos) manifestó haber participado en al menos una actividad creativa. De este modo, se aprecia un cierto desconocimiento del término «vacaciones creativas», así como la dificultad que implica separar el turismo creativo del cultural. Este comportamiento es comprensible teniendo en cuenta que ambas formas de turismo tienen el objetivo común de conocer la cultura, aunque el creativo implique una mayor interacción con el sitio y sus habitantes.

La Figura 9 expone las únicas actividades creativas que fueron seleccionadas, de las cuales el tour gastronómico fue la de mayor atracción. Seguidamente, se ubican las visitas a actividades productivas como labores de marisqueo, pesca o industriales; y a talleres de artistas y artesanos. El interés por la gastronomía de Galicia vuelve a destacar mediante la realización de talleres; y por último con muy poca presencia aparecen los talleres de música y artesanía.

\section{Figura 9}

\section{ACTIVIDADES CREATIVAS REALIZADAS POR LOS ENTREVISTADOS}

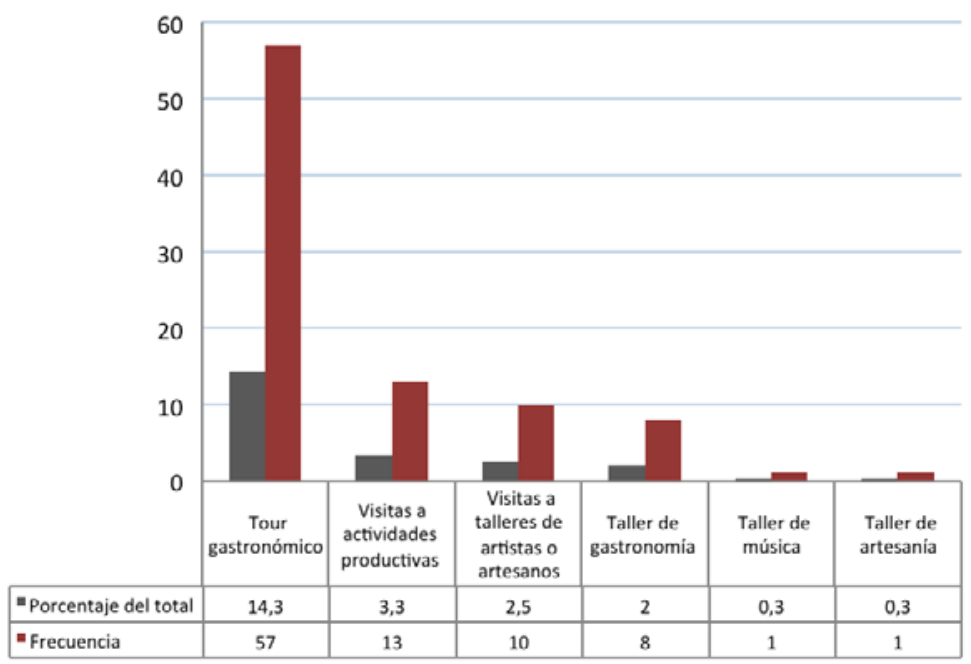


Asimismo, se analizó la influencia de algunos aspectos del perfil de los entrevistados en la preferencia por estas cuatro actividades creativas. Para ello se valoraron las siguientes variables: el sexo, la edad, el nivel de estudios, la renta, la ocupación y el gasto. La Tabla 1 exhibe los valores de significación de la prueba Chi-cuadrado. El taller de gastronomía fue preferido por las mujeres, en esta actividad no hubo participación masculina. Por su parte, en las visitas a talleres de artistas influyeron la edad y el nivel de estudios. De este modo, el $50 \%$ de las personas que realizaron esta actividad tenían más de 50 años y estudios de máster o doctorado.

Tabla 1

VALORES DE SIGNIFICACIÓN DE LA PRUEBA CHI-CUADRADO SEGÚN ACTIVIDAD CREATIVA Y VARIABLE DEL PERFIL

\begin{tabular}{ccccc}
\hline & \multicolumn{3}{c}{ Prueba de Chi-cuadrado (sig.asintótica bilateral) } \\
\hline Variable & $\begin{array}{c}\text { tour } \\
\text { gastronómico }\end{array}$ & $\begin{array}{c}\text { Visitas } \\
\text { actividades } \\
\text { productivas }\end{array}$ & $\begin{array}{c}\text { Visitas } \\
\text { artistas }\end{array}$ & $\begin{array}{c}\text { Taller de } \\
\text { gastronomía }\end{array}$ \\
\hline Sexo & 0,233 & 0,058 & 0,520 & 0,026 \\
Edad & 0,147 & 0,413 & 0,001 & 0,784 \\
Nivel de estudios & 0,770 & 0,364 & 0,027 & 0,096 \\
Renta & 0,644 & 0,128 & 0,497 & 0,231 \\
Ocupación & 0,437 & 0,768 & 0,458 & 0,831 \\
Gasto & 0,481 & 0,660 & 0,750 & 0,407 \\
\hline
\end{tabular}

\subsection{Satisfacción e imagen}

De forma general los visitantes se sintieron satisfechos con la visita a Santiago de Compostela, la media fue de 8,47 (escala de 1 a 10), alcanzando un valor mínimo de 3 y un máximo de 10. Sin embargo, más del $50 \%$ otorgó una puntuación de 9 o 10 . Este resultado es similar al recogido por ATLAS, donde también se obtuvo un valor por encima de 8 (Richards, 2008). A su vez, la Tabla 2 muestra los aspectos que fueron considerados para evaluar la imagen que tenían los visitantes sobre la autenticidad del paisaje y los elementos culturales del destino (en este caso, en una escala de 1 a 5).

Para conocer si existían diferencias significativas entre las medias correspondientes a cada uno de estos elementos se realizó una t de Student. Antes de realizar la prueba se verificaron los supuestos de normalidad y homogeneidad de varianza, comprobando que se cumplían en todos los casos. Como resultado se obtuvo que las diferencias entre los valores de las medias que recoge la Tabla 2 son significativas, con tres excepciones. Ellas son: entre hospitalidad de la población y gastronomía (sig. 0,172), entre distinción cultural y museos (sig. 0,346), y entre región multicultural y costumbres (sig. 0,212). Por tanto, estos aspectos son apreciados de forma similar. De este modo, se puede decir que la arquitectura histórica es el aspecto mejor valorado; seguido del ambiente del lugar, y en tercer lugar y al mismo nivel, la hospitalidad de la población y la gastronomía. Mientras que, Santiago no fue considerado como un sitio moda, recibiendo este aspecto la peor puntuación. 


\section{Tabla 2 \\ ASPECTOS RELACIONADOS CON LA AUTENTICIDAD DEL PAISAJE}

\begin{tabular}{lcc}
\hline Aspecto & Media $^{\mathbf{a}}$ & Desviación típica \\
\hline Arquitectura histórica & 4,49 & 0,715 \\
Ambiente lugar & 4,33 & 0,722 \\
Hospitalidad población & 4,20 & 0,824 \\
Gastronomía regional & 4,13 & 0,869 \\
Distinción cultural & 4,01 & 0,889 \\
Museos y atracciones culturales & 3,96 & 0,913 \\
Diversidad lingüística & 3,77 & 1,074 \\
Región multicultural & 3,57 & 1,060 \\
Costumbres y tradiciones & 3,48 & 1,035 \\
Sitio de moda & 3,14 & 1,166 \\
\hline
\end{tabular}

Nota: ${ }^{\text {Escala de } 1 \text { a } 5 .}$

\section{CONCLUSIONES}

El visitante en Santiago de Compostela se corresponde con el tradicional turista cultural de edad madura. Como muestran los datos de los últimos años del Observatorio Turístico de Santiago, no se observa el incremento de jóvenes en el volumen total visitantes, tendencia que se manifiesta en el turismo mundial, tal y como señalan ATLAS y la Organización Mundial del Turismo. Este hecho hace reflexionar en la necesidad de reformular la oferta de la ciudad para captar al mercado juvenil, así como diseñar una política de promoción que permita alcanzar a este segmento. La proporción de mujeres es mayor que la de los hombres, comportamiento similar al de los resultados de ATLAS y de otros autores. En cuanto a la procedencia, los mercados internacionales más importantes son: Alemania, Francia, el Reino Unido y Portugal, aunque este último más asociado al excursionismo. Sin embargo, se mantiene la elevada dependencia del mercado español, el cual representa más de la mitad del total de visitantes.

Como ocurre en la mayoría de los destinos, las vacaciones constituyen la principal motivación para visitar Santiago de Compostela. A pesar de ser un destino cultural, los visitantes que viajan exclusivamente para disfrutar de un atractivo o asistir a un evento (turistas motivados culturalmente) suponen una pequeña porción. Está claro que la mayor parte de los turistas viajan por motivos recreacionales y de placer, en detrimento de experiencias profundas de aprendizaje, aunque la mayoría se involucre en mayor o menor medida en actividades culturales. De este resultado se desprende la necesidad de adecuar la oferta cultural tradicional a esta búsqueda de experiencias recreativas que demandan los visitantes en la actualidad. En este sentido, hay que comentar que aunque se ha incrementado su popularidad en los últimos años, las visitas a los museos tienen un menor protagonismo que en el resto de destinos culturales estudiados por ATLAS, lo cual puede ser un indicio de la necesidad de revitalizar la oferta museística según las exigencias de la actual demanda. 
Para los visitantes la arquitectura histórica sigue siendo el aspecto más novedoso del paisaje compostelano, pero también destaca la alta valoración otorgada al ambiente del lugar, a la gastronomía y a la hospitalidad de la población. Son precisamente estos últimos elementos los que muchos destinos culturales están aprovechando para revitalizarse y adecuarse a estas nuevas expectativas de los visitantes. De este modo, proliferan iniciativas para conocer la esencia de las ciudades en vez de las áreas turísticas, experimentar los estilos de vida y costumbres de la mano de los propios residentes, o aprender oficios tradicionales junto a los propios artesanos. Así, los visitantes señalaron como principal atracción la realización del tour gastronómico, aunque también mencionaron otras actividades como las visitas a actividades productivas, a talleres de artistas y artesanos, o la realización de talleres. Sin embargo, en Santiago no es posible profundizar en la dimensión que está teniendo en la actualidad el turismo creativo. Esto se debe a que este tipo de actividades están concebidas más bien para grupos; y a que la celebración de eventos, especialmente gastronómicos, se realiza de forma puntal. Este trabajo muestra cómo el patrimonio histórico de Santiago de Compostela sigue siendo su principal reclamo turístico, pero cómo al mismo tiempo se demanda un ambiente creativo y más actual, en torno a actividades más recreativas y participativas.

\section{REFERENCIAS}

ALDREY, J.A., SANTOS, X.M. Y PAZOS M. (2009): «Caso de estudio. Santiago de Compostela». En Materiales didácticos de la asignatura Practicum II. Diplomatura en Turismo de la Universitat Oberta de Catalunya. Barcelona. Universitat Oberta de Catalunya, pp. 1-44.

ATLAS (2015): «The ATLAS Cultural Tourism Project: Summary of Research Results». Disponible en http://www.tram-research.com/atlas/previous.htm

BACKER, E. (2007): «VFR travel. An Examination of the Expenditures of VFR Travellers and their Hosts», Current Issues in Tourism, vol. 10, n 4, pp. 366-377.

CHAMORRO, R. (2008): «Una revolución en el turismo gracias a las TIC», Bit, $\mathrm{n}^{\circ}$ 170, pp. 30-33.

DOMÍNGUEZ, T. y ARAÚJO, N, (2012): «El fenómeno 2.0 en el sector turístico. El caso de Madrid 2.0», Pasos, vol. 10, n 3, pp. 225-238.

ESCUDERO, L. (2013): «La imagen urbana de Santiago de Compostela (España), un estudio de su representación pública, mediática, promocional y artística», Boletín de la Asociación de Geógrafos Españoles, nº 62, pp. 265-294.

EXCELTUR (2013): «UrbanTUR2012. Monitor de Competitividad Turística de los Destinos Urbanos Españoles». Disponible en http://www.exceltur.org/urbantur/

HERNÁNDEZ-MOGOLLÓN, J.M.; DI-CLEMENTE, E.; FOLGADO-FERNÁNDEZ, J.A. y CAMPÓN-CERRO, A.M. (2013): «La imagen del destino como elemento determinante en la definición del perfil del turista cultural actual. El caso de Plasencia en España», En TMS Conference Series. Competitividade, marketing e tecnologias de informação e comunicação no turismo. Universidad de Algarve. Escola Superior de Gestão, hotelería e turismo, pp. 159-172. 
ISAAC, R., (2008): Understanding the Behaviour of Cultural Tourists. Towards a Classification of Dutch Cultural Tourists. Dissertation. Breda, NHTV International Higher Education Breda.

LOIS, R. y LÓPEZ, L. (2012): «El Camino de Santiago: una aproximación a su carácter polisémico desde la geografía cultural y el turismo», Documents d'Anàlisi Geogràfica, vol. 58, no 3, pp. 459-479.

MCKERCHER, B. y DU CROS, H. (2002): Cultural Tourism: The Partnership Between Tourism and Cultural Heritage Management. New York, Haworth Press.

MCKERCHER, B. y DU CROS, H. (2003): «Testing a Cultural Tourism Typoly». International Journal of Tourism Research, $\mathrm{n}^{\circ}$ 5, pp. 45-58. Doi: 10.1002/jtr.417.

MINISTERIO DE EDUCACIÓN, CULTURA Y DEPORTE (2013): «CulturaBase. Turismo cultural». Disponible en http://www.mcu.es/culturabase/cgi/um?M=/t7/p7/ a $2005 / \& O=$ pcaxis $\& N=\& L=0$

OBSERVATORIO TURÍSTICO DE SANTIAGO (2014): Datos de la demanda turística del 2008-2012. Santiago de Compostela, Centro de Estudios e Investigaciones Turísticas. Universidad de Santiago de Compostela.

OLIVIERA, C.; DE MAN, A.; GUERREIRO, S. y FARIA, L. (2013): «Novos desafíos de mercado para as agências de viagens», En III Congreso Internacional de Turismo, Barcelos, 18 y 19 de octubre de 2013.

OMT (2011): «AM Reports Volumen 2 'La Fuerza del Turismo Joven'». Disponible en http:// www2.unwto.org/es/publication/am-reports-volumen-2-la-fuerza-del-turismo-joven

PÉREZ GUILARTE, Y. (2014): «Nuevas tendencias del turismo cultural. El papel de la investigación turística en ciudades patrimoniales: los casos de Santiago de Compostela y La Habana Vieja». (Tesis doctoral). MINERVA. Repositorio Institucional de la Universidad de Santiago de Compostela. Disponible en http://dspace.usc.es/ handle/10347/11872

RICHARDS, G. (2008): «ATLAS Cultural Tourism Survey. Summary Report 2007».Disponible en http://www.tram-research.com/atlas/ATLAS\%20Cultural\%20Tourism\%20 Survey\%202007.PDF

RICHARDS, G. (2012): «Tourism, Creativity and Creative Industries». En Creativity and Creative Industries in Challenging Times Conference. NHTV Breda, Noviembre. Disponible en https://www.academia.edu/Documents/in/Creative_Tourism?page $=3$

RICHARDS, G. y RAYMOND, C. (2000): «Creative Tourism» ATLAS News n ${ }^{\circ} 23$, pp.16-20. Disponible en http://www.atlas-euro.org/

RICHARDS, G. y QUEIRÓS C. (2005): «ATLAS Cultural Tourism Project. 2004 Survey Report». Disponible en http://www.atlas-webshop.org/epages/61492534.sf/en_ GB/?ObjectPath=/Shops/61492534/Products/ATL_00046

SANTOS, X.M. (2006): «El camino de Santiago: turistas y peregrinos hacia Compostela», Cuadernos de Turismo, $\mathrm{n}^{\circ} 18$, pp. 135-150.

SANTOS, X.M. y LOIS, R. (2011): «El camino de Santiago en el contexto de los nuevos turismos», Estudios Turísticos, n 89 , pp. 95-116.

UNESCO (2006): «Towards Sustainable Strategies for Creative Tourism. Discussion Report of the Planning Meeting for 2008 International Conference on Creative Tourism». Disponible en http://unesdoc.unesco.org/images/0015/001598/159811E.pdf 
WURZBURGUER, R.; AAGESON, T.; PATTAKOS, A. y PRATT, S. (2008): Creative Tourism. A global conversation. How to Provide Unique Creative Experiences for Travelers Worldwide. Santa Fe, Sunston Press. 